\title{
Dielectric Properties and Characterisation of the Superionic Phase of $\left[\mathrm{C}\left(\mathrm{NH}_{2}\right)_{3}\right]_{2} \mathrm{SbCl}_{5}{ }^{*}\left[\mathrm{C}\left(\mathrm{NH}_{2}\right)_{3}\right] \mathrm{Cl}(\mathrm{GHCA})$
}

\author{
J. Zaleski ${ }^{\mathrm{a}}$, G. Bator ${ }^{\mathrm{b}}$, and R. Jakubas ${ }^{\mathrm{b}}$ \\ a Institute of Chemistry, University of Opole, 45-951 Opole, Oleska 48, Poland \\ b Institute of Chemistry, University of Wroclaw, 50-383 Wrockaw, Joliot Curie 14, Poland
}

Z. Naturforsch. 50a, 888-892 (1995); received February 20, 1995

\begin{abstract}
GHCA undergoes four phase transitions at $T_{c 1}=402 \mathrm{~K}, T_{c 2}=373 \mathrm{~K}, T_{c 3}=162 \mathrm{~K}$, and $T_{c 4}=146 \mathrm{~K}$. Below $T_{c 3}$ it possesses pyroelectric properties with the spontaneous polarization vector $\left(P_{\mathrm{s}}\right)$ in the ac plane and the maximum along the $c$ axis equal to $8 \mu \mathrm{C} / \mathrm{m}^{2}$. Dielectric dispersion studies of $\mathrm{GHCA}$ show that the main dielectric dispersion connected probably with collective motions of chlorine ions is above $1 \mathrm{GHz}$. For the phase transition at $T_{c 2}$ to a superionic phase the thermal dilatation and electric conductivity were measured. The anomalies of the electric conductivity at $T_{c 2}$ and $T_{c 1}$ were observed with large values of $\sigma\left(10^{-3} \mathrm{~S} / \mathrm{m}\right)$ above $T_{c 1}$. The guanidinium cations above $T_{c 2}$, besides reorientational motions, undergo slow self diffusion.
\end{abstract}

Key words: Guanidinium, chloroantimonate, phase transition, DSC, dilatometric, pyroelectric, electric conductivity.

\section{Introduction}

The alkylammonium halogenoantimonates (III) and bismuthates (III) have recently attracted considerable attention $[1-4]$ because many members of the group possess rich sequences of phase transitions, some of them to polar phases. On substitution of alkylammonium cations by guanidinium $\left(\mathrm{C}\left(\mathrm{NH}_{2}\right)_{3}^{+}\right)$ones, two new salts have been obtained: $\left[\mathrm{C}\left(\mathrm{NH}_{2}\right)_{3}\right]_{2} \mathrm{SbCl}_{5}$ * $\mathrm{C}\left(\mathrm{NH}_{2}\right)_{3} \mathrm{Cl}$ (GHCA) [5] and $\left[\mathrm{C}\left(\mathrm{NH}_{2}\right)_{3}\right]_{3} \mathrm{Sb}_{2} \mathrm{Cl}_{9}{ }^{*}$ $0.9 \mathrm{H}_{2} \mathrm{O}$ (GNCA) [6].

They are molecular ionic salts. GNCA (space group $\mathrm{C} 2 / \mathrm{c})$ possesses an anionic sublattice composed of two-dimensional polyanionic $\left(\mathrm{Sb}_{2} \mathrm{Cl}_{9}^{3-}\right)_{n}$ layers built of corner sharing $\mathrm{SbCl}_{6}^{3-}$ octahedra. There are two crystallographically non-equivalent cations: one situated together with water molecule inside cavities formed inside the polyanionic layers the other one between the layers [7].

In GNCA two phase transitions have been found at $T_{c 1}=364 \mathrm{~K}$ and $T_{c 2}=105 \mathrm{~K}$. The transition at $T_{c 1}$ is probably connected with dehydration of GNCA. The transition at $T_{c 2}$, found from dielectric studies, is connected with continuous slowing down of reorientations of one of the two non-equivalent cations [6].

GHCA crystallizes in $\mathrm{P} 2_{1} / \mathrm{c}$ space group. It is built of one dimensional polyanionic $\left(\mathrm{SbCl}_{5}^{2-}\right)_{n}$ chains, ex-

Reprint requests to Dr. Jacek Zaleski. tended along the $c$ direction, and isolated chlorine anions $\mathrm{Cl}^{-}$situated, together with guanidinium cations, between polyanionic chains. The guanidinium cations are connected to chlorines by electrostatic interactions and $\mathrm{N}-\mathrm{H} \ldots \mathrm{Cl}$ hydrogen bonds [8].

In GHCA three phase transitions have been found at $370 \mathrm{~K}, 162 \mathrm{~K}$ and at $146 \mathrm{~K}$. The two low temperature transitions were suggested to be of the displacive type, and the high temperature one of the order disorder type. Dielectric properties of GHCA are substantially different from that of GNCA. It is most probably connected with the different construction of the anionic sublattice. In GHCA a large anisotropy of the dielectric properties was found with maximal permittivity along the $c$ direction [5]. A sharp decrease of the real part of the complex eléctric permittivity at $146 \mathrm{~K}$ suggests the presence of a polar phase. ${ }^{1} \mathrm{H}$ NMR studies of the molecular motions in GHCA showed that below $146 \mathrm{~K}$ the cationic sublattice is rigid on the NMR time scale. The onset of reorientations of cations around their $\mathrm{C}_{3}$ axis takes place between 200 and $370 \mathrm{~K}$. Above $370 \mathrm{~K}$ a plastic phase, characterised by simultaneous tumbling and self diffusion of guanidinium cations, was found [9].

In the present work we show dielectric dispersion and pyroelectric studies of GHCA in the low temperature region and characterise GHCA in the high temperature region by DSC, electric conductivity and thermal dilatation. 


\section{Experimental}

Single crystals of GHCA were prepared as described in [5]. DSC experiments were carried out on a Perkin Elmer DSC-7 calorimeter with a coding/heating rate of $5-20 \mathrm{~K} / \mathrm{min}$.

The linear thermal expansion was measured by a thermomechanical analyser Perkin-Elmer TMS-2. The studies were performed in the heating and cooling mode at rates of $0.1-0.2 \mathrm{~K} / \mathrm{min}$. The anomalies were reproducible within $10 \%$ for each sample. The accuracy of the thermal expansion determination is about $2 \%$. The samples had dimensions of about $4-5 \mathrm{~mm}$ along the $a, b$, and $c$ axes.

The measurements of the pyroelectric current and charge were performed under short-circuit conditions during linear heating or cooling of the sample (with a rate of $1 \mathrm{~K} / \mathrm{min}$ ) on a Keithley 617 type electrometer.

The silver painted area of the samples for the pyroelectric studies was about $17 \mathrm{~mm}^{2}$ perpendicular to the $c$-axis and $9 \mathrm{~mm}^{2}$ perpendicular to tha $a$-axis. The temperature was controlled by a UNIPAN Temperature Controller type 660 to an accuracy $\pm 0.1 \mathrm{~K}$. The reproducibility of the pyroelectric current was better than $20 \%$ for all samples, and the experimental error of $\Delta P_{\mathrm{s}}$ was smaller than $0.1 \mu \mathrm{C} / \mathrm{m}^{2}$.

The electrical conductivity was measured on a Keithley 617 type electrometer for DC conditions. Gold electrodes were deposited on the crystal used in the measurements. The dimensions of samples were similar to those used in pyroelectric studies.

For the dielectric measurements, samples of dimensions $(8 \times 2 \times 0.5) \mathrm{mm}^{3}$ were cut perpendicularly to the $c$-axis. The plates were silver painted. The complex electric permittivity, $\varepsilon^{*}=\varepsilon^{\prime}-i \varepsilon^{\prime \prime}$, was measured by an HP 4191A Impedance Analyser in the frequency range from $30 \mathrm{MHz}$ to $900 \mathrm{MHz}$. The temperature was stabilized and controlled by a UNIPAN Temperature Controller type 660 . The over-all error of the real part of the complex electric permittivity was smaller than $10 \%$.

\section{Results}

\section{DSC}

In Fig. 1 DSC curves during cooling and heating of GHCA in the temperature range $340-410 \mathrm{~K}$ are presented. Below room temperature no heat anomalies were observed. Above room temperature, on heating

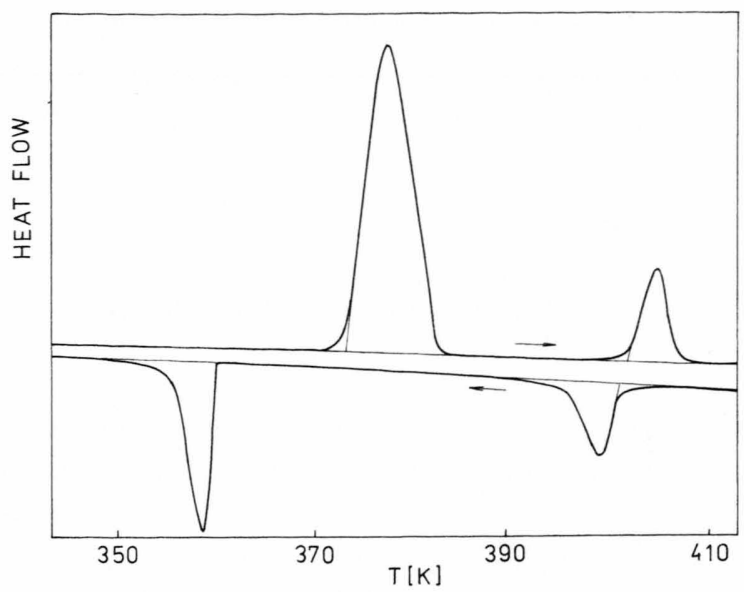

Fig. 1. DSC curves of GHCA during cooling and heating runs above room temperature.

Table 1. Transition temperatures, enthalpies and entropies of GHCA.

\begin{tabular}{lll}
\hline$T$ & $\Delta H[\mathrm{~kJ} / \mathrm{mol}]$ & $\Delta S\left[\mathrm{~J} / \mathrm{K}^{*} \mathrm{~mol}\right]$ \\
\hline 373 & 16.6 & 44.4 \\
402 & 2.3 & 5.7 \\
426 & 17.8 & 41.8 \\
\hline
\end{tabular}

three distinct anomalies in the heat flow were observed at $373 \mathrm{~K}, 402 \mathrm{~K}$ and $426 \mathrm{~K}$. GHCA melts at $426 \mathrm{~K}$, whereas the two other anomalies correspond to the solid-solid phase transitions at $T_{c 1}=402 \mathrm{~K}$ and at $T_{c 2}=373 \mathrm{~K}$. The transition at $T_{c 2}$ has a large thermal hysteresis of $13 \mathrm{~K}$. It corresponds to that found at $370 \mathrm{~K}$ from NMR measurements. The shift of the temperature seems to be dependent on the quality of the crystals.

The transition enthalpies and entropies are presented in Table 1. It should be noted that the transition entropy at $T_{c 2}$ is comparable to that of melting. This indicates that at $T_{c 2}$ process connected with significant increase of disorder of one of the sublattices takes place. Results of NMR studies show that for this process the cationic sublattice is responsible. It may be explained as a pre-melting process. The transition enthalpy at $T_{c 2}$ during the cooling run depends on the time the sample is kept above $T_{c 2}$. It varies from $1 / 3$ of the initial enthalpy to zero when the annealing time increases. This effect is probably connected with the time GHCA needs to reach thermal equilibrium. Slow transition kinetics depending on the size of sample grains were also observed for guanidinium iodide [10]. 

lows:

The phase transition sequence of GHCA is as fol-

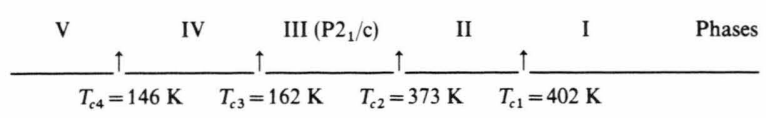

\section{Phase Transitions below Room Temperature}

\section{a) Pyroelectric}

The typical temperature dependence of the spontaneous polarization $\left(P_{\mathrm{s}}\right)$ and the pyroelectric current (I) of the GHCA crystal along the $c$ axis is shown in Fig. 2. In the high temperature phase III, far from the phase transition, the polarization is about zero. On lowering the temperature below $T_{c 3}, P_{\mathrm{s}}$ increases continuously. The transition at $T_{c 4}$ is accompanied by a jump in the polarization of the order of $0.3-0.8 \mu \mathrm{C} / \mathrm{m}^{2}$, depending on the sample. The pyroelectric anomaly is fully reversible during cooling and heating, which confirms its pyroelectric nature.

The pyroelectric effect was also measured along the $a$ - and $b$-axes. The changes of $P_{\mathrm{s}}$ along the $a$-axis are similar to those along the $c$-axis but about three times lower. Along the $b$-axis no pyroelectric effect was observed. The observed pyroelectric anomaly in the $b c$ plane suggests that the transition at $T_{c 3}$ takes place to a phase with a space group of point symmetry $m$ (most probably from $\mathrm{P} 2_{1} / \mathrm{c}$ to $\mathrm{Pc}$ ). In $\mathrm{Pc}$ space group the polar vector may have components in two perpendicular directions in the ac plane, which finds confirmation in our experimental results.

The observed values of spontaneous polarization are relatively small. Attempts of monodomenisation of the sample by applying an external electric field $E$ up to $10^{5} \mathrm{~V} / \mathrm{m}$ resulted, however, in large electric conductivity, which did not vanish even after 10 hours of short circuited sample. It did not allow to determine whether $P_{s}$ is reversible under DC electric field.

\section{b) Dielectric Dispersion}

In Fig. 3 the temperature dependence of $\varepsilon_{c}^{\prime}$ recorded at $30,153.5$, and $840.8 \mathrm{MHz}$ is shown. Measurements of the complex electric permittivity in the frequency range $30 \mathrm{MHz}-0.9 \mathrm{GHz}$ did not show any relaxation process neither near the phase transitions at $T_{c 3}$ and $T_{c 4}$ nor in the phases III and V. The increase of the electric permittivity in phase IV is observed for frequencies lower than $1 \mathrm{MHz}$ [5] but not visible above

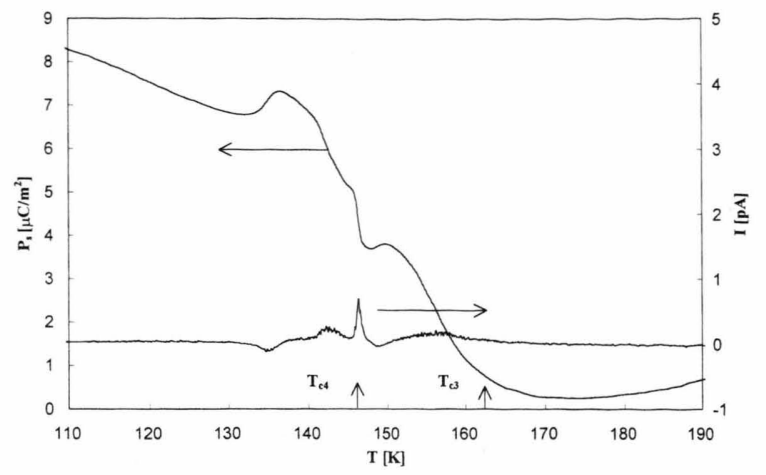

Fig. 2. Temperature dependence of the spontaneous polarization and pyroelectric current of GHCA near the phase transitions at $T_{c 3}=162 \mathrm{~K}$ and $T_{c 4}=146 \mathrm{~K}$.

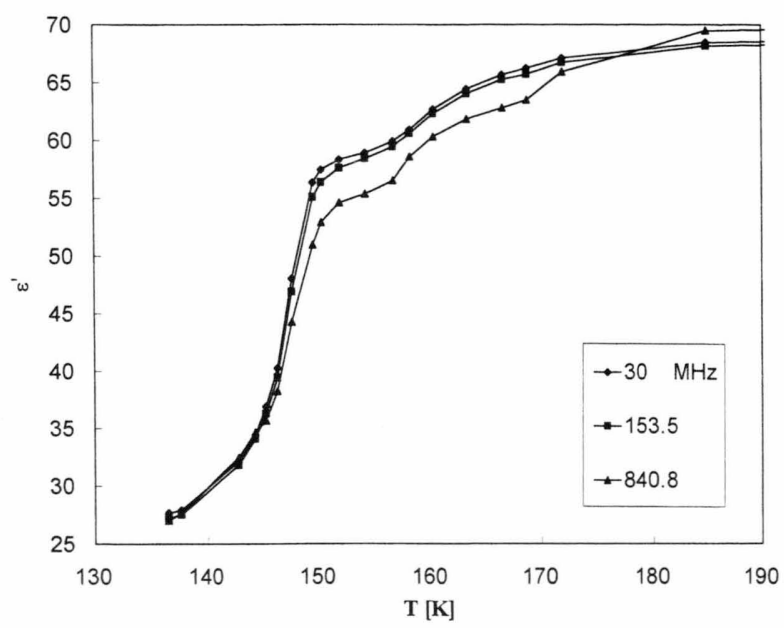

Fig. 3. Temperature dependence of the real part of the electric permittivity of GHCA measured along the $c$ axis at 30, 153.5 and $840.8 \mathrm{MHz}$ in the low temperature region.

$30 \mathrm{MHz}$. It suggests the presence of low frequency dielectric dispersion of non-Debye nature in phase IV.

The main dielectric dispersion, connected most probably with displacive collective motions of chlorine ions, is expected to occur in a sub-millimetre range.

\section{Phase Transitions above Room Temperature}

\section{a) Dilatometric}

In Fig. $4 \mathrm{a}-\mathrm{c}$ the temperature dependence of the thermal dilatation along the $a, b$ and $c$ axes near $T_{c 2}$ is presented. Thermal dilatation measurements close 

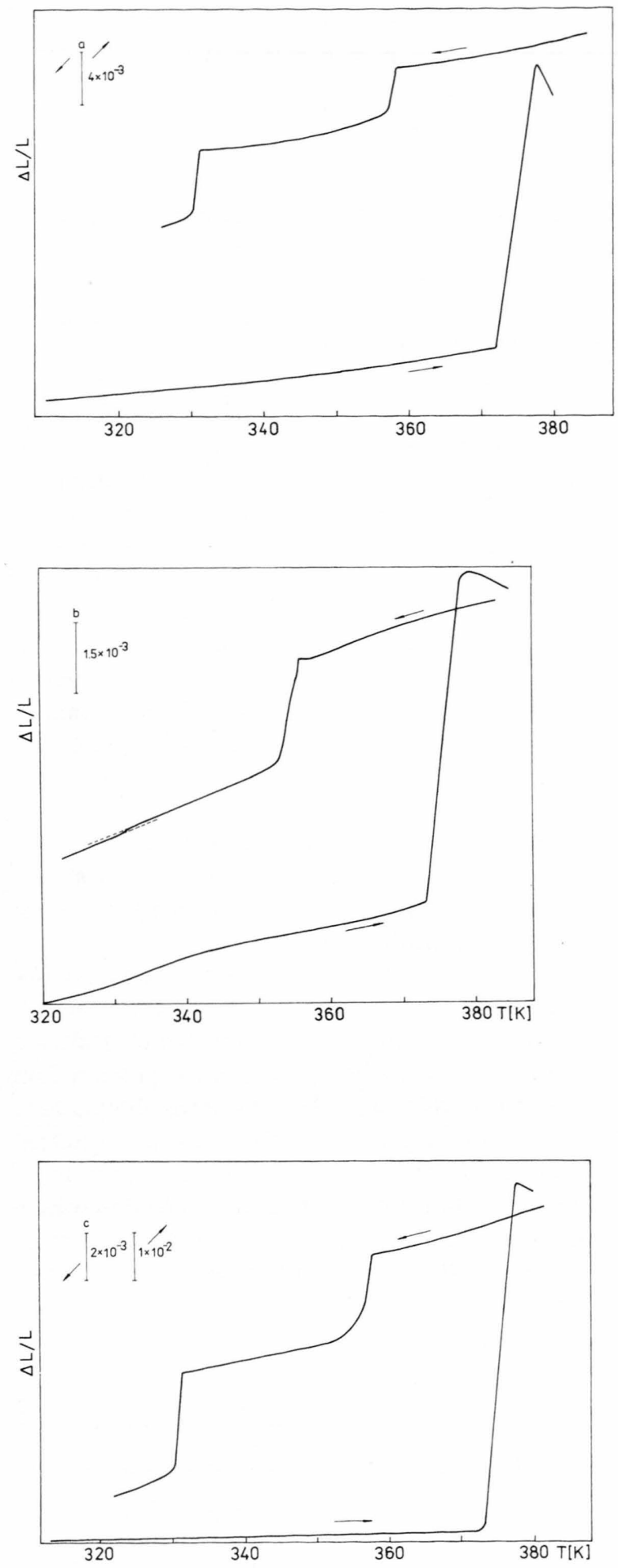

Fig. $4 \mathrm{a}-\mathrm{c}$. Temperature dependence of the thermal dilatation of GHCA along the $a, b$, and $c$ directions near the phase transition at $T_{c 2}=373 \mathrm{~K}$.

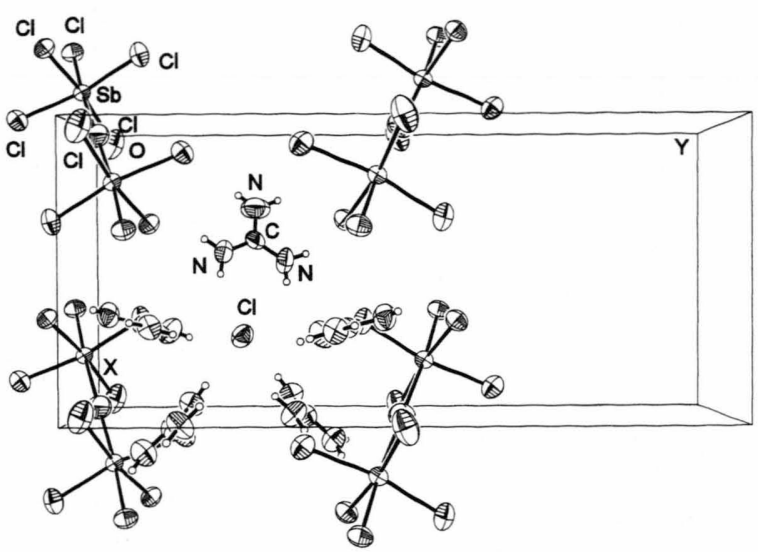

Fig. 5. Crystal structure of GHCA as seen along the $c$ direction. Four one dimensional polyanionic $\left(\mathrm{SbCl}_{5}^{3-}\right)_{n}$ chains built of $\mathrm{SbCl}_{6}^{3-}$ octahedra are extended perpendicular to the figure plane forming infinite tunnels. The guanidinium $\mathrm{C}\left(\mathrm{NH}_{2}\right)_{3}^{+}$cations together with isolated $\mathrm{Cl}^{-}$anions are located inside the tunnels.

to $T_{c 1}$ were not performed since the crystal softens mechanically on passing $T_{c 2}$.

On increasing temperature an anomaly of the thermal dilatation along all crystallographic directions is observed. The dilatation along the $c$ direction $(\approx 7 \%)$ is about one order of magnitude larger than those along the $a$ and $b$ directions. The increase of the length of the sample along the $c$ direction may be connected with partial melting of the lattice at $T_{c 2}$, which finds confirmation in results of DSC as well as ${ }^{1} \mathrm{H}$ NMR studies. The NMR experiments proved the presence of cation tumbling and self diffusion above $T_{c 2}$ [9].

The polyanionic $\left(\mathrm{SbCl}_{5}^{3-}\right)_{n}$ chains are extended along the $c$ axis. Each four chains form infinite tunnels along the $c$ axis where the cations and isolated chlorine ions are located (Fig. 5). It is therefore, not surprising that during the melting of the cationic sublattice the elongation of the sample is maximum along the $c$ direction.

On decreasing temperature two anomalies of thermal dilatation along all crystallographic directions at 358 and $331 \mathrm{~K}$ show up. On increasing the temperature, a second time, again one anomaly at $373 \mathrm{~K}$ is observed. There are two explanations possible: either on decreasing the temperature below $T_{c 2}$, GHCA enters an intermediate phase III' before returning to the initial phase III, or on decreasing temperature phase II is supercooled and the thermal dilatation anomaly reflects a decrease of cationic reorientation 


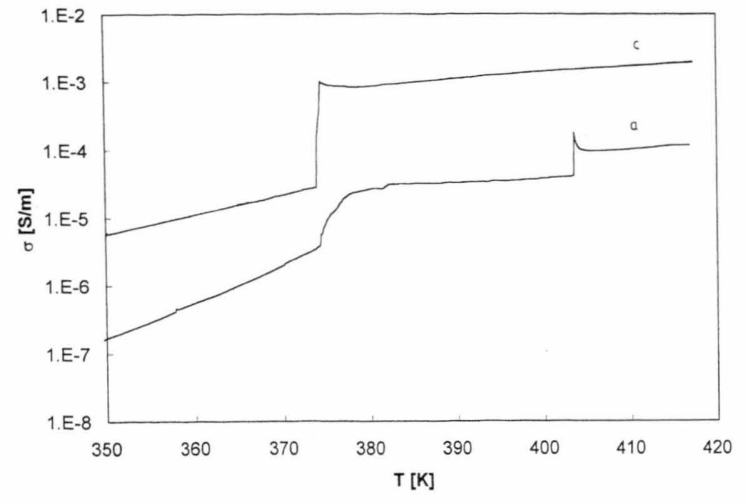

Fig. 6. Temperature dependence of the DC conductivity of GHCA near the phase transitions at $T_{c 2}=373 \mathrm{~K}$ and $T_{c 1}=402 \mathrm{~K}$ along the $c$ and $a$ axes.

at $331 \mathrm{~K}$. It should be noted that around $330 \mathrm{~K}$ no anomaly of the heat flow was observed on cooling in DSC experiments.

The decrease of lengths along all directions on cooling below $T_{c 2}$ is smaller than the initial increase at $T_{c 2}$. This may be explained by the significant polycrystalliziation of the initial single crystal which takes place at $T_{c 2}$. Such effect was observed in the X-ray diffraction studies where at $T_{c 2}$ the intensities of all reflections decreased to zero [8].

\section{b) Electric Conductivity}

In Fig. 6 the temperature dependence of the DC conductivity measured along the $c$ and $a$ axes near $T_{c 1}$ and $T_{c 2}$ is presented. In the temperature dependence of $\sigma_{c}(T)$ only one anomaly is visible at $T_{c 2}$, whereas in $\sigma_{a}(T)$ there are two anomalies at $T_{c 2}$ and $T_{c 1}$. A large anisotropy of the electric conductivity in the ac plane should be noted. The large conductivity above $T_{c 2}$ corroborates the presence of a superionic phase. The activation energy along the $c$ direction in phase III is estimated to be $1.2 \mathrm{eV}$, while in phases I and II it is
$0.3 \mathrm{eV}$. The activation energy along the a direction are $1.6,0.1$, and $0.3 \mathrm{eV}$ in phases III, II, and I, respectively.

\section{Conclusions}

The properties of GHCA are substantially different from those of GNCA. This is connected with different anionic sublattices. GNCA is built of polyanionic two-dimensional layers whereas the anionic sublattice of GHCA is composed of one dimensional polyanionic chains and isolated chlorine ions.

The physical properties of GHCA are determined by the presence of isolated chlorine ions in the polyanionic vacancies connected to guanidinium cations by moderately strong $(\mathrm{N}-\mathrm{H} \ldots \mathrm{Cl})$ hydrogen bonds.

The large anisotropy of the dielectric properties in the low temperature region, with a maximum of $\varepsilon^{\prime}$ along the $c$ direction, may be explained by collective shifts of the $\mathrm{Cl}^{-}$ions. Such shifts of isolated $\mathrm{Cl}^{-}$ions are facilitated by the presence of long, one-dimensional polyanionic vacancies. Below $T_{c 3}$ the librations of chlorine anions gradually freeze, with a large decrease of amplitudes at $T_{c 4}$, which results in a dramatic decrease of the electric permittivity and the appearance of spontaneous polarization below $T_{c 3}$. Lack of dielectric dispersion in the microwave region excludes the presence of the order-disorder mechanism of the phase transitions at $T_{c 3}$ and at $T_{c 4}$.

Phase transition at $T_{c 2}$ to a superionic phase is associated with a large transition entropy, which may be rationalised by assuming that at this temperature the network of hydrogen bonds connecting guanidinium cations to $\mathrm{Cl}$ ions breaks. As a result we observe slow diffuse motion of guanidinium cations and resultant large values of electric conductivity. The maximum of the conductivity is found along the $c$ direction, where the motions of cations are expected to take place most easily. The transition at $T_{c 1}$ is not well understood at present.
[1] R. Jakubas and L. Sobczyk, Phase Transitions 20, 163 (1990).

[2] J. Mroz and R. Jakubas, Ferroelectrics 118, 29 (1991).

[3] M. Iwata and Y. Ishibashi, Ferroelectrics 135, 283 (1992).

[4] H. Ishikara, K. Watanabe, A. Iwata, K. Yamada, Y. Kinoshita, T. Okuda, V. G. Krishnan, S. Dou, and A. Weiss, Z. Naturforsch. 47a, 65 (1992).

[5] J. Zaleski and R. Jakubas, Z. Naturforsch. 49a, 709 (1994).
[6] J. Zaleski, R. Jakubas, G. Bator, and J. Baran, J. Mol Struct. 325, 95 (1994).

[7] J. Zaleski and A. Pietraszko, Z. Naturforsch. 49a, 895 (1994).

[8] J. Zaleski and A. Pietraszko, J. Mol. Struct. 327, 287 (1994).

[9] Z. Pająk and J. Zaleski, Solid State Commun. 91, 821 (1994).

[10] S. Gima, Y. Furukawa, and D. Nakamura, Ber. Bunsenges. Phys. Chem. 88, 939 (1984). 